\title{
Corrigendum
}

\section{Genetic Influences on Hormonal Markers of Chronic HPA Function in Human Hair - CORRIGENDUM}

\section{E. M. Tucker-Drob et al.}

DOI: https://doi.org/10.1017/S0033291716003068, first published online by Cambridge University Press 19 January 2017

Key words: Cortisol, gene-age interaction, hair hormones, hypothalamic-pituitary-adrenal axis, quantitative genetics.

In the above article (Tucker-Drob et al. (2017) the authors mistakenly reported $90 \%$ in place of $95 \%$ confidence intervals. The article has been corrected below to report 95\% confidence intervals in Table 1, Table 3, and Table S1 of the online supplement. In addition, Figure 1 of the article has been updated so that the gray bands reflect $95 \%$ confidence intervals. All parameter estimates and p values originally reported are correct. All inferences and conclusions are therefore unchanged.

Table 1. Descriptive statistics, phenotypic correlations, and cross-twin correlations

\begin{tabular}{|c|c|c|c|c|c|}
\hline & Males & & Females & & \\
\hline \multicolumn{6}{|c|}{ Means (+/-1 SD range) } \\
\hline cortisol (pg/mg) & \multicolumn{2}{|l|}{$3.39(1.08,10.69)$} & \multicolumn{2}{|l|}{$2.95(0.92,9.44)$} & \\
\hline cortisone (pg/mg) & \multicolumn{2}{|l|}{$6.50(2.28,12.90)$} & \multicolumn{2}{|l|}{$5.21(1.42,11.40)$} & \\
\hline Age (years) & \multicolumn{2}{|l|}{$12.08(9.44,14.72)$} & \multicolumn{2}{|l|}{$12.65(9.80,15.48)$} & \\
\hline \multicolumn{6}{|c|}{ Phenotypic Correlations (95\% CI's) } \\
\hline cortisol-cortisone & \multicolumn{2}{|l|}{$.54(.42, .67)$} & \multicolumn{2}{|l|}{$.57(.46, .67)$} & \\
\hline SES-cortisol & \multicolumn{2}{|l|}{$.02(-.11, .16)$} & \multicolumn{2}{|l|}{$.04(-.06, .13)$} & \\
\hline SES-cortisone & \multicolumn{2}{|l|}{$-.06(-.17, .04)$} & \multicolumn{2}{|l|}{$.00(-.09, .10)$} & \\
\hline age-cortisol & \multicolumn{2}{|l|}{$.01(-.07, .09)$} & \multicolumn{2}{|l|}{$.23(.14, .32)$} & \\
\hline age-cortisone & \multicolumn{2}{|l|}{$.06(-.03, .15)$} & \multicolumn{2}{|l|}{$.30(.22, .37)$} & \\
\hline \multicolumn{6}{|c|}{ Cross-Twin Correlations (95\% CI's) } \\
\hline & $\mathrm{MZ}$ & $\mathrm{DZ}$ & $\mathrm{MZ}$ & $\mathrm{DZ}$ & DZ Opposite-Sex \\
\hline cortisol-cortisol & $.74(.51, .97)$ & $.53(.31, .76)$ & $.58(.41, .76)$ & $.34(.14, .54)$ & $.26(.10, .43)$ \\
\hline cortisone-cortisone & $.56(.37, .75)$ & $.47(.30, .65)$ & $.49(.33, .66)$ & $.36(.17, .56)$ & $.22(.01, .43)$ \\
\hline cortisol-cortisone & $.44(.25, .63)$ & $.21(.03, .39)$ & $.22(.08, .36)$ & $.32(.17, .48)$ & $.06(-.08, .19)$ \\
\hline
\end{tabular}

Note. Sample means and +/-1 SD range were computed after cortisol and cortisone were square root and log transformed, respectively, and winsorized. We then exponentiated (cortisol) or squared (cortisone) the calculated values in order to return them to their original metric. Cross-twin and phenotypic correlations were estimated from models that controlled for sexspecific linear effects of age. For cross-twin and phenotypic correlations cortisone and cortisol were square root and log transformed, respectively, and winsorized. 
Table 3. Unstandardized Parameter Estimates from Sex, Age, and SES Moderation Models

\begin{tabular}{|c|c|c|c|c|c|c|c|c|c|c|c|c|}
\hline \multirow{2}{*}{$\begin{array}{l}\text { Parameter } \\
\text { Variance in Cortisone }\end{array}$} & \multicolumn{3}{|c|}{ Preferred Sex Moderation Model } & \multicolumn{3}{|c|}{ Preferred Age Moderation Model } & \multicolumn{3}{|c|}{$\begin{array}{l}\text { Preferred SES Model (No } \\
\text { Moderation) }\end{array}$} & \multicolumn{3}{|c|}{ Full SES Moderation } \\
\hline & Estimate & $(95 \% C I)$ & p-value & Estimate & $(95 \% C I)$ & p-value & Estimate & $(95 \% C I)$ & p-value & Estimate & $(95 \% C I)$ & p-value \\
\hline Main $A$ effect $\left(\mathrm{A}_{\mathrm{CNO}}\right)$ & .68 & $(.46, .90)$ & $<.001$ & .42 & $(.03, .80)$ & .04 & .63 & $(.37, .88)$ & $<.001$ & .70 & $(.52, .89)$ & $<.001$ \\
\hline$A \times$ Sex Interaction $\left(\mathrm{A}_{\mathrm{CN} 1}\right)$ & -.05 & $(-.28, .17)$ & .64 & - & - & - & - & - & - & - & - & - \\
\hline$A \times$ Age Interaction $\left(\mathrm{A}_{\mathrm{CN} 1}\right)$ & - & - & - & .03 & $(-.02, .09)$ & .25 & - & - & - & - & - & - \\
\hline$A \times \mathrm{SES}$ Interaction $\left(\mathrm{A}_{\mathrm{CN} 1}\right)$ & - & - & - & - & - & - & - & - & - & -.13 & $(-.26, .01)$ & .08 \\
\hline Main $C$ effect $\left(\mathrm{C}_{\mathrm{CNO}}\right)$ & .35 & $(-.02, .71)$ & .06 & .67 & $(.37, .97)$ & $<.001$ & .41 & $(.10, .72)$ & .009 & .26 & $(-.12, .64)$ & .17 \\
\hline$C \times$ Sex Interaction $\left(\mathrm{C}_{\mathrm{CN} 1}\right)$ & .19 & $(-.03, .40)$ & .08 & - & - & - & - & - & - & - & - & - \\
\hline$C \times$ Age Interaction $\left(\mathrm{C}_{\mathrm{CN} 1}\right)$ & - & - & - & -.05 & $(-.11, .02)$ & .14 & - & - & - & - & - & - \\
\hline$C \times \mathrm{SES}$ Interaction $\left(\mathrm{C}_{\mathrm{CN} 1}\right)$ & - & - & - & - & - & - & - & - & - & .12 & $(.02, .22)$ & .02 \\
\hline Main $E$ effect $\left(\mathrm{E}_{\mathrm{CN} 0}\right)$ & .64 & $(.56, .72)$ & $<.001$ & .57 & $(.44, .71)$ & $<.001$ & .63 & $(.54, .73)$ & $<.001$ & .61 & $(.53, .69)$ & $<.001$ \\
\hline$E \times \operatorname{Sex}$ Interaction $\left(\mathrm{E}_{\mathrm{CN} 1}\right)$ & -.13 & $(-.27, .01)$ & .07 & - & - & - & - & - & - & - & - & - \\
\hline$E \times$ Age Interaction $\left(\mathrm{E}_{\mathrm{CN} 1}\right)$ & - & - & - & .02 & $(-.01, .05)$ & .13 & - & - & - & - & - & - \\
\hline$E \times \mathrm{SES}$ Interaction $\left(\mathrm{E}_{\mathrm{CN} 1}\right)$ & - & - & - & - & - & - & - & - & - & .04 & $(-.04, .12)$ & .37 \\
\hline Main Sex effect & .63 & $(39, .87)$ & $<.001$ & .62 & $(.38, .85)$ & $<.001$ & .55 & $(.32, .79)$ & $<.001$ & .56 & $(.32, .80)$ & $<.001$ \\
\hline Main Age effect & .08 & $(.06, .11)$ & $<.001$ & .08 & $(.06, .11)$ & $<.001$ & .08 & $(.05, .10)$ & $<.001$ & .08 & $(.05,10)$ & $<.001$ \\
\hline Age $\times$ Sex Interaction & -.06 & $(-.11,<-.01)$ & .04 & -.06 & $(-.11,-.01)$ & .03 & -.04 & $(-.09, .01)$ & .14 & -.04 & $(-.09, .01)$ & .11 \\
\hline Main SES effect & - & - & - & - & - & - & -.01 & $(-.09, .07)$ & .84 & -.01 & $(-.08, .08)$ & .92 \\
\hline Race (Hispanic) & .31 & $(.09, .53)$ & .01 & .30 & $(.08, .52)$ & .01 & .21 & $(-.01, .43)$ & .06 & .21 & $(-.01, .44)$ & .06 \\
\hline Race (African American) & .33 & $(-.04, .71)$ & .08 & .33 & $(-.05, .71)$ & .08 & .26 & $(-.11, .64)$ & .17 & .28 & $(-.10, .67)$ & .15 \\
\hline Race (Other) & .11 & $(-.12, .34)$ & .34 & .10 & $(-.13, .33)$ & .40 & .09 & $(-.13, .32)$ & .42 & .10 & $(-.13, .32)$ & .40 \\
\hline \multicolumn{13}{|l|}{ Variance in Cortisol } \\
\hline Main $A$ effect $\left(\mathrm{A}_{\mathrm{C} 0}\right)$ & .79 & $(.65, .94)$ & $<.001$ & .79 & $(.44,1.13)$ & $<.001$ & .72 & $(.52, .92)$ & $<.001$ & .75 & $(.63, .86)$ & $<.001$ \\
\hline$A \times$ Age Interaction $\left(\mathrm{A}_{\mathrm{C} 1}\right)$ & - & - & - & -.02 & $(-.08, .04)$ & .49 & - & - & - & - & - & - \\
\hline$A \times \mathrm{SES}$ Interaction $\left(\mathrm{A}_{\mathrm{C} 1}\right)$ & - & - & - & - & - & - & - & - & - & -.03 & $(-.20, .14)$ & .77 \\
\hline Main $C$ effect $\left(\mathrm{C}_{\mathrm{C} 0}\right)$ & -.06 & $(-1.36,1.24)$ & .93 & .69 & $(.32,1.06)$ & $<.001$ & .32 & $(-.03, .66)$ & .07 & .09 & $(-.24, .43)$ & .59 \\
\hline$C \times$ Age Interaction $\left(\mathrm{C}_{\mathrm{C} 1}\right)$ & - & - & - & -.13 & $(-.17,-.08)$ & $<.001$ & - & - & - & - & - & - \\
\hline $\mathrm{C} \times \mathrm{SES}$ Interaction $\left(\mathrm{C}_{\mathrm{C} 1}\right)$ & - & - & - & - & - & - & - & - & - & .24 & $(.08, .41)$ & .004 \\
\hline Main $E$ effect $\left(\mathrm{E}_{\mathrm{C} 0}\right)$ & .58 & $(.51, .66)$ & $<.001$ & .69 & $(.54, .84)$ & $<.001$ & .58 & $(.50, .66)$ & $<.001$ & .57 & $(.50, .65)$ & $<.001$ \\
\hline$E \times$ Age Interaction $\left(E_{C 1}\right)$ & - & - & - & -.02 & $(-.05, .01)$ & .16 & - & - & - & - & - & - \\
\hline$E \times \mathrm{SES}$ Interaction $\left(\mathrm{E}_{\mathrm{C} 1}\right)$ & - & - & - & - & - & - & - & - & - & -.02 & $(-.11, .07)$ & .66 \\
\hline Main Sex effect & .57 & $(.31, .83)$ & $<.001$ & .54 & $(.29, .79)$ & $<.001$ & .55 & $(.32, .79)$ & $<.001$ & .54 & $(.28, .80)$ & $<.001$ \\
\hline Main Age effect & .05 & $(.02, .08)$ & $<.001$ & .05 & $(.02, .08)$ & $<.001$ & .05 & $(.02, .08)$ & .001 & .05 & $(.02, .08)$ & $<.001$ \\
\hline Age $\times$ Sex Interaction & -.07 & $(-.11,-.02)$ & .009 & -.06 & $(-.11,-.01)$ & .01 & -.05 & $(-.10,-.01)$ & .02 & -.05 & $(-.10,-.01)$ & .03 \\
\hline Main SES effect & - & - & - & - & - & - & .04 & $(-.04, .12)$ & .38 & .02 & $(-.06, .10)$ & .58 \\
\hline
\end{tabular}




\begin{tabular}{|c|c|c|c|c|c|c|c|c|c|c|c|c|}
\hline \multirow{2}{*}{$\begin{array}{l}\text { Parameter } \\
\text { Variance in Cortisone }\end{array}$} & \multicolumn{3}{|c|}{ Preferred Sex Moderation Model } & \multicolumn{3}{|c|}{ Preferred Age Moderation Model } & \multicolumn{3}{|c|}{$\begin{array}{l}\text { Preferred SES Model (No } \\
\text { Moderation) }\end{array}$} & \multicolumn{3}{|c|}{ Full SES Moderation } \\
\hline & Estimate & $(95 \% C I)$ & p-value & Estimate & $(95 \% C I)$ & p-value & Estimate & $(95 \% C I)$ & p-value & Estimate & $(95 \% C I)$ & p-value \\
\hline Race (Hispanic) & .02 & $(-.16, .20)$ & .83 & .04 & $(-.15, .22)$ & .70 & .02 & $(-.17, .20)$ & .86 & .01 & $(-.17, .19)$ & .90 \\
\hline Race (African American) & .81 & $(.37,1.26)$ & $<.001$ & .83 & $(.40,1.27)$ & $<.001$ & .66 & $(.19,1.13)$ & .01 & .68 & $(.21,1.16)$ & .01 \\
\hline Race (Other) & -.08 & $(-.33, .17)$ & .51 & -.08 & $(-30, .15)$ & .50 & -.10 & $(-.36, .15)$ & .43 & -.09 & $(-.35, .17)$ & .50 \\
\hline \multicolumn{13}{|l|}{ Cortisone with Cortisol } \\
\hline Main $A$ Correlation $\left(\mathrm{r}_{\mathrm{a} 0}\right)$ & .78 & $(.51,1.04)$ & $<.001$ & .74 & $(-.05,1.52)$ & .07 & .73 & $(.39,1.07)$ & $<.001$ & .65 & $(.40, .91)$ & $<.001$ \\
\hline $\mathrm{r}_{\mathrm{A}} \times$ Age Interaction $\left(\mathrm{r}_{\mathrm{a} 1}\right)$ & - & - & - & $<.01$ & $(-.12, .13)$ & .96 & - & - & - & - & - & - \\
\hline $\mathrm{r}_{\mathrm{A}} \times \mathrm{SES}$ Interaction $\left(\mathrm{r}_{\mathrm{a} 1}\right)$ & - & - & - & - & - & - & - & - & - & -.03 & $(-.45, .39)$ & .89 \\
\hline Main $C$ Correlation $\left(\mathrm{r}_{\mathrm{c} 0}\right)$ & 2.76 & $(-59.63,65.11)$ & .93 & .58 & $(.01,1.15)$ & .05 & .23 & $(-.81,1.27)$ & .67 & 1.33 & $(-.24,2.90)$ & .10 \\
\hline $\mathrm{r}_{\mathrm{C}} \times$ Age Interaction $\left(\mathrm{r}_{\mathrm{c} 1}\right)$ & - & - & - & -.10 & $(-.31, .11)$ & .35 & - & - & - & - & - & - \\
\hline $\mathrm{r}_{\mathrm{C}} \times \mathrm{SES}$ Interaction $\mathrm{r}_{\mathrm{c} 1}$ ) & - & - & - & - & - & - & - & - & - & -.99 & $(-2.40, .41)$ & .17 \\
\hline Main E Correlation $\left(\mathrm{r}_{\mathrm{e} 0}\right)$ & .42 & $(.28, .56)$ & $<.001$ & .19 & $(-.07, .44)$ & .15 & .40 & $(.24, .56)$ & $<.001$ & .41 & $(.26, .55)$ & $<.001$ \\
\hline $\mathrm{r}_{\mathrm{E}} \times$ Age Interaction $\left(\mathrm{r}_{\mathrm{e} 1}\right)$ & - & - & - & .06 & $(.02, .10)$ & .001 & - & - & - & - & - & - \\
\hline $\mathrm{r}_{\mathrm{E}} \times \mathrm{SES}$ Interaction $\left(\mathrm{r}_{\mathrm{e} 1}\right)$ & - & - & - & - & - & - & - & - & - & $<.01$ & $(-.20, .19)$ & .99 \\
\hline
\end{tabular}

Note. $95 \% \mathrm{CI}=95 \%$ confidence interval. p-value = two-tailed probability of Type-I error. $A=$ additive genetic; $C=$ shared environment; $E=$ non-shared environment. Results are presented for preferred (based on model comparisons) age and sex moderation models. Results are presented for the preferred age, sex, and SES moderation models based on model comparisons. The preferred sex model allowed for moderation of only cortisone ACE estimates, while the preferred age moderation model estimated age moderation for cortisol, cortisone, and the ACE correlations. The preferred SES model did not allow for moderation by SES of any parameters; however, results are also provided for a model that allowed SES to moderate cortisol, cortisone, and the $A C E$ correlations. Sex was effects coded (female =-.5, male =.5) such that the main effects parameters of $A$, $C$, and $E$ represent population-mean effects (assuming an equal sex distribution in the population) and interaction effects of sex by $A, C$, and $E$ represent the sex difference in the corresponding parameter value. Age was centered at 8 years of age to reflect the lowest observed integer value in the sample. Thus the main effects parameters of $A, C$, and $E$ represent model-implied biometric effects at age 8 years, and interaction effects of age by $A, C$, and $E$ represent the difference in the corresponding parameter value for each additional year of age. 

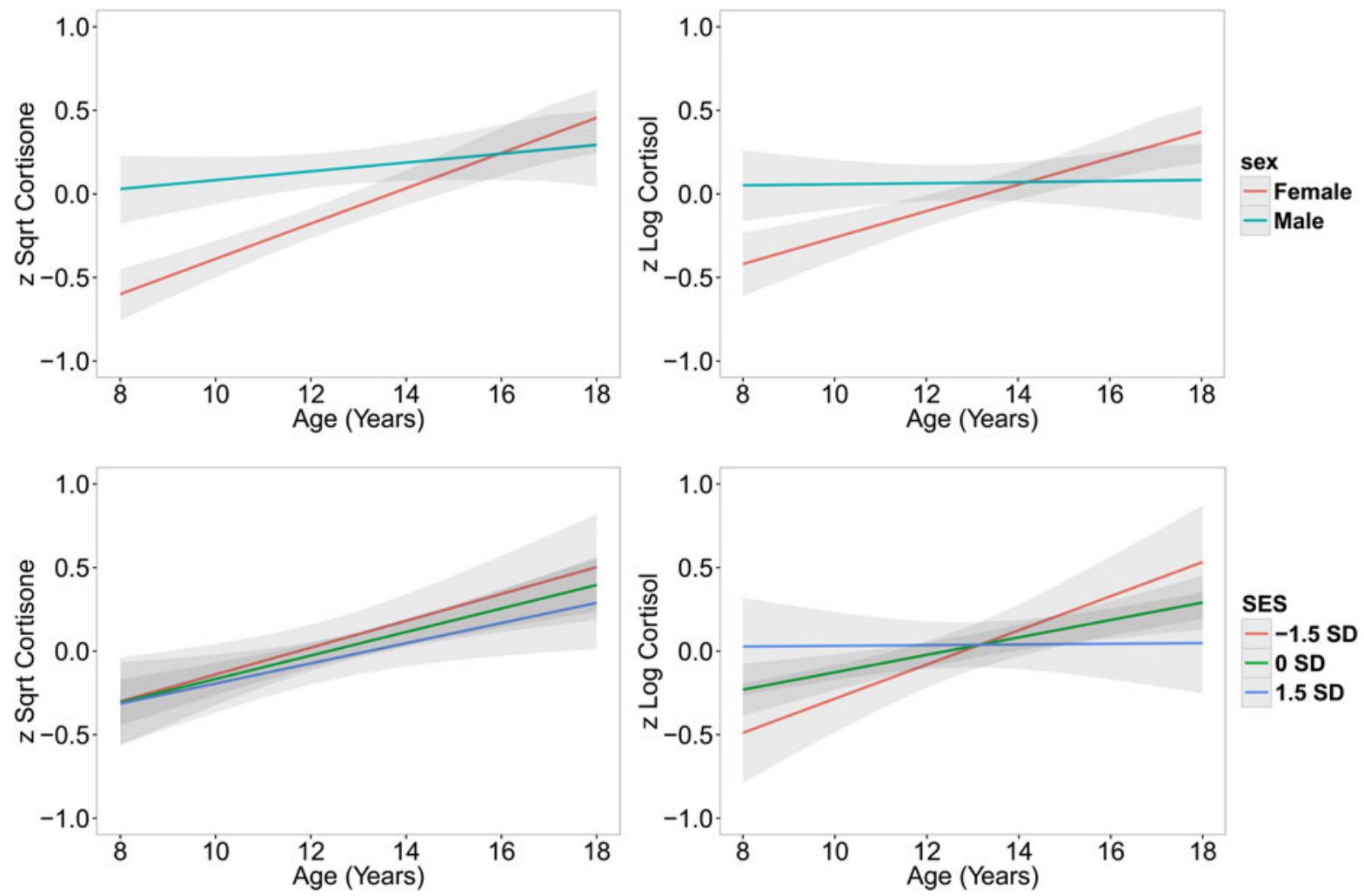

Fig. 1.

\section{Supplementary Material}

The supplementary material for this article can be found at https://doi.org/10.1017/S0033291717001325.

\section{Reference}

Tucker-Drob E.M. et al. (2017). Genetic Influences on Hormonal Markers of Chronic HPA Function in Human Hair, Psychological Medicine doi:10.1017/S0033291716003068 